\title{
Growth Rates and Explosions in Sandpiles
}

\author{
Anne Fey $\cdot$ Lionel Levine $\cdot$ Yuval Peres
}

Received: 29 January 2009 / Accepted: 29 November 2009 / Published online: 9 December 2009

(C) The Author(s) 2009. This article is published with open access at Springerlink.com

\begin{abstract}
We study the abelian sandpile growth model, where $n$ particles are added at the origin on a stable background configuration in $\mathbb{Z}^{d}$. Any site with at least $2 d$ particles then topples by sending one particle to each neighbor. We find that with constant background height $h \leq 2 d-2$, the diameter of the set of sites that topple has order $n^{1 / d}$. This was previously known only for $h<d$. Our proof uses a strong form of the least action principle for sandpiles, and a novel method of background modification.

We can extend this diameter bound to certain backgrounds in which an arbitrarily high fraction of sites have height $2 d-1$. On the other hand, we show that if the background height $2 d-2$ is augmented by 1 at an arbitrarily small fraction of sites chosen independently at random, then adding finitely many particles creates an explosion (a sandpile that never stabilizes).
\end{abstract}

Keywords Abelian sandpile · Bootstrap percolation - Dimensional reduction · Discrete Laplacian · Growth model · Least action principle

\section{Introduction}

In this paper we consider the abelian sandpile model as a growth model in the integer lattice $\mathbb{Z}^{d}$. The model starts from a stable background configuration in which each site $x$ has

\footnotetext{
A. Fey

Delft Institute of Applied Mathematics, Delft University of Technology, Delft, The Netherlands e-mail: a.c.fey-denboer@tudelft.nl

L. Levine ( $\square)$

Department of Mathematics, Massachusetts Institute of Technology, Cambridge, MA 02139, USA

e-mail: levine@math.mit.edu

url: http://math.mit.edu/ levine

Y. Peres

Theory Group, Microsoft Research, Redmond, WA 98052, USA

e-mail: peres@microsoft.com
} 

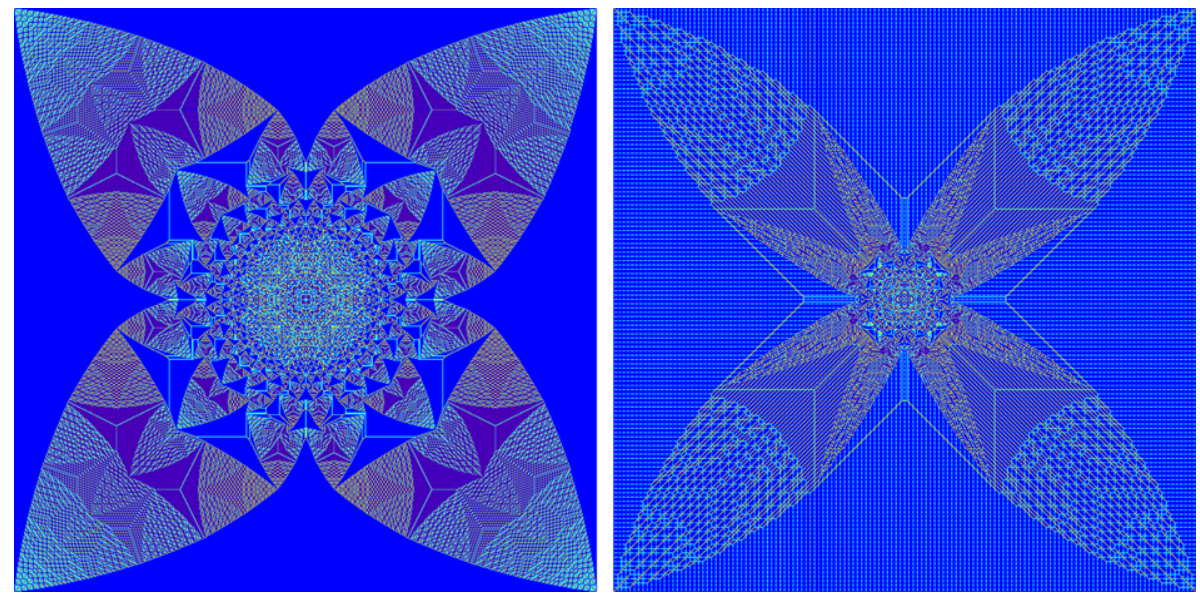

Fig. 1 (Color online) Left: Stable sandpile of $n=2 \cdot 10^{5}$ particles in $\mathbb{Z}^{2}$ on background height $h=2$. Right: Sandpile of $n=15000$ particles in $\mathbb{Z}^{2}$ on background height 3, except every fifth row and column has background height 2 . In both cases, the set $T_{n}$ is a square. Color scheme: sites colored blue have 3 particles, turquoise 2 particles, yellow 1 particle, red 0 particles

a pile of $\sigma(x) \leq 2 d-1$ particles. To this background, $n$ particles are added at the origin. Typically, $n$ is large. We stabilize this configuration by toppling every unstable site; that is, every site with at least $2 d$ particles gives one particle to each of its neighbors, until there are no more unstable sites. For more information on the abelian sandpile model, also known as the chip-firing game, see $[1-3,11]$.

To keep things simple in this introduction, we will enumerate the sites in $\mathbb{Z}^{d}$ (say, in order of increasing distance from the origin, breaking ties arbitrarily) and perform topplings one by one in discrete time: At each time step, if there are any unstable sites, then the smallest unstable site topples. All of our results hold also for the more general toppling procedures discussed in Sect. 2.

Let $T_{n}=T_{n, d, \sigma}$ be the set of sites that topple (Fig. 1). Since these sets are nested, $T_{1} \subseteq$ $T_{2} \subseteq \cdots$, it is natural to view them as a growth model, with $n$ playing the role of a time parameter. We distinguish between two extreme cases. If $T_{n}$ is finite for all $n$, we say that $\sigma$ is robust. In this case we are interested in the growth rate, i.e. in how the diameter of $T_{n}$ grows with $n$.

At the other extreme, if $T_{n}=\mathbb{Z}^{d}$ for some $n$, then every site topples infinitely often. Otherwise, some site $x \in \mathbb{Z}^{d}$ must finish toppling before all of its neighbors do; since each neighbor topples at least once after $x$ finishes toppling, $x$ receives $2 d$ additional particles and must topple again.

If $T_{n}=\mathbb{Z}^{d}$ for some $n$, then we say that $\sigma$ is explosive, and it is exploding when the $n$ particles are added. (In [7], the term 'not stabilizable' was used for 'exploding,' and 'metastable' for 'explosive.') The simplest example of an explosive background is $\sigma(x)=2 d-1$ for all $x$, to which the addition of a single extra particle causes every site in $\mathbb{Z}^{d}$ to topple infinitely many times.

We remark that an intermediate behavior is possible, when $T_{n}$ is infinite for a finite $n$, but $T_{n} \neq \mathbb{Z}^{d}$ for all $n$. An example is the background with 3 particles at every site on the $x$-axis in $\mathbb{Z}^{2}$, and 2 particles at every other site. Adding one particle at the origin produces an infinite avalanche of topplings, but each site topples only finitely many times. This example 
shows that exploding is a strictly stronger condition than having an infinite avalanche of topplings.

The papers [6] and [10] investigated the case of a robust constant background of $h \leq$ $2 d-2$ particles at every site. In the regime $h<d$, the diameter of $T_{n}$ grows like $n^{1 / d}$; the best known bounds can be found in [10, Theorem 4.1]. In the case $h=2 d-2$, the set $T_{n}$ is a cube for every $n$, and an upper bound for the radius is $n$ [6, Theorem 4.1]. No proof was found for a better upper bound, even though simulations clearly indicated a growth rate proportional to $n^{1 / d}$.

In this paper we complete the picture by deriving an upper bound of order $n^{1 / d}$ on the diameter of $T_{n}$ for all $h \leq 2 d-2$, and even for some backgrounds arbitrarily close to $2 d-1$. We first correct a gap in the proof of the outer bound of [10, Theorem 4.1], which we thank Haiyan Liu for pointing out to us. Then we use this theorem together with a new technique of "background modification" to extend the bounds to higher values of $h$.

Throughout the paper, we will typically use the symbol $\sigma$ to indicate a stable background configuration, and $\eta$ to indicate an arbitrary (possibly unstable) configuration. We use $\bar{h}$ to denote the constant configuration $\sigma(x) \equiv h$, and we denote a single particle at the origin by $\delta_{o}$. Write

$$
Q_{r}=\left\{x \in \mathbb{Z}^{d}: \max \left|x_{i}\right| \leq r\right\}
$$

for the cube of side length $2 r+1$ centered at the origin in $\mathbb{Z}^{d}$. Let $\omega_{d}$ be the volume of the unit ball in $\mathbb{R}^{d}$.

Our main result, proved in Sect. 3, is the following.

Theorem 1.1 Fix integers $d \leq h \leq 2 d-2$, and let $T_{n, d, \bar{h}}$ be the set of sites in $\mathbb{Z}^{d}$ that topple during the stabilization of $\bar{h}+n \delta_{o}$. Then for any $\epsilon>0$, we have

$$
T_{n, d, \bar{h}} \subset Q_{r}
$$

for all sufficiently large $n$, where

$$
r=\frac{d+\epsilon}{2 d-1-h}\left(\frac{n}{\omega_{d}}\right)^{1 / d} .
$$

In the case $d=h=2$, Theorem 1.1 gives a bound of $\frac{2+\epsilon}{\sqrt{\pi}} \sqrt{n} \approx 1.13 \sqrt{n}$ on the radius of the square of sites that topple. Large scale simulations by David Wilson indicate that the actual radius is approximately $0.75 \sqrt{n}$.

It is natural to ask what happens when the background height $h$ exceeds $2 d-2$. While the background $\overline{2 d-1}$ is explosive, our next result shows that there exist robust backgrounds in which an arbitrarily high proportion of sites have $2 d-1$ particles. For $m \geq 1$, let

$$
\Lambda(m)=\left\{x \in \mathbb{Z}^{d}: m \not x_{i} \text { for all } 1 \leq i \leq d\right\} .
$$

Thus $\Lambda(m)$ is a union of cubes of side length $m-1$. The following theorem generalizes the case $h=2 d-2$ of Theorem 1.1, which corresponds to the case $m=1$.

Theorem 1.2 For any $m \geq 1$, the background

$$
\sigma=\overline{2 d-2}+1_{\Lambda(m)}
$$


Fig. 2 (Color online) An exploding sandpile started from $n=5000$ particles in $\mathbb{Z}^{2}$.

Background height is 2 except for sites in the lattice generated by $(1,10)$ and $(10,1)$, which have background height 3 .

Unstable sites are colored black

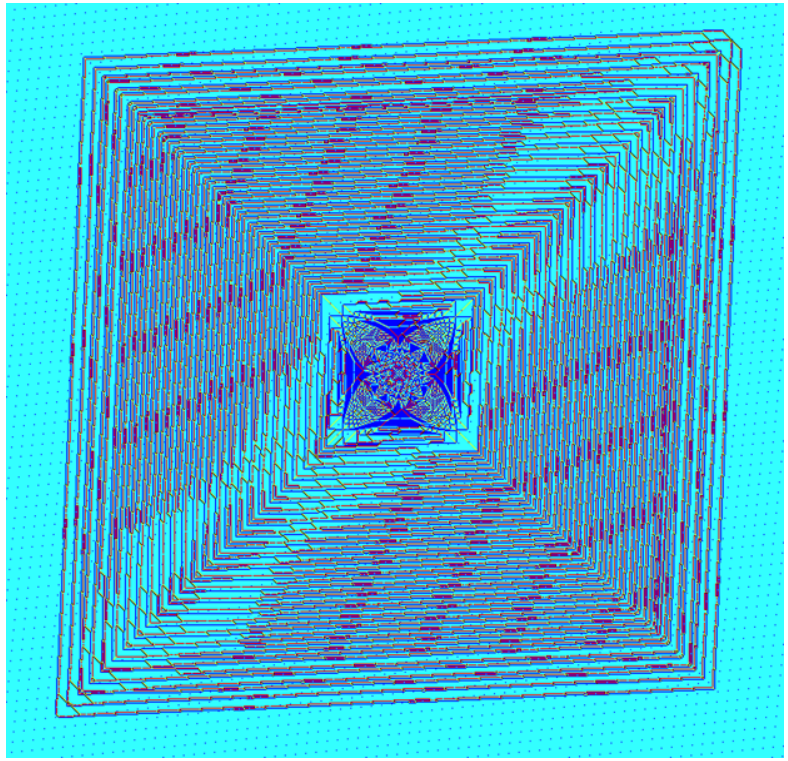

is robust on $\mathbb{Z}^{d}$. Moreover, writing $T_{n, d, \sigma}$ for the set of sites in $\mathbb{Z}^{d}$ that topple during the stabilization of $\sigma+n \delta_{o}$, then for any $\epsilon>0$, we have

$$
T_{n, d, \sigma} \subset Q_{r}
$$

for all sufficiently large $n$, where

$$
r=m(d+\epsilon)\left(\frac{n}{\omega_{d}}\right)^{1 / d} .
$$

On the basis of this theorem, one might guess that $2 d-1$ is the critical density below which a background is robust and above which it is explosive. Our next two results show that this is not the case. Starting from background height $2 d-2$, we can destroy robustness by adding extra particles on an arbitrarily sparse lattice $L \subset \mathbb{Z}^{d}$ (Fig. 2).

Proposition 1.3 Let $\mathbf{x}_{i}=\left(x_{i 1}, \ldots, x_{i d}\right)$ for $i=1, \ldots, d$ be linearly independent vectors in $\mathbb{Z}^{d}$ satisfying $\operatorname{gcd}\left(x_{1 j}, \ldots, x_{d j}\right)=1$ for all $j=1, \ldots$, d. Let $L=\mathbb{Z} \mathbf{x}_{1}+\cdots+\mathbb{Z} \mathbf{x}_{d}$. Then the background $\overline{2 d-2}+1_{L}$ on $\mathbb{Z}^{d}$ is explosive.

Comparing Theorem 1.2 and Proposition 1.3, we see that the geometry of the extra particles plays a more important role in determining robustness than the density of sites at which particles are added. In particular, the fact that $L$ intersects every hyperplane in $\mathbb{Z}^{d}$ that is parallel to one of the coordinate hyperplanes, while $\Lambda(m)$ does not, plays a key role in the proofs.

As our next result shows, the lattice structure is not essential in Proposition 1.3. We can also produce an explosive background by adding particles at rare random sites.

Proposition 1.4 Fix $\epsilon>0$, and let $(\beta(x))_{x \in \mathbb{Z}^{d}}$ be independent Bernoulli random variables with $\mathbb{P}(\beta(x)=1)=\epsilon$. With probability 1 , the background $\overline{2 d-2}+\beta$ on $\mathbb{Z}^{d}$ is explosive. 
Our proofs make extensive use of the abelian property of the abelian sandpile model, which we state and generalize in the next section.

\section{Least Action Principle}

We begin by recalling the notion of toppling procedure defined in [8]. This formalism includes most of the natural ways to topple, including: discrete time parallel updates, in which all unstable vertices topple simultaneously; toppling in nested volumes, in which we successively stabilize larger and larger finite regions of $\mathbb{Z}^{d}$; and Markov toppling in continuous time, in which each site has a Poisson clock and attempts to topple whenever its clock rings. The technical details of the toppling procedures are tangential to our main argument, so the reader may wish to skim them and move on to the "least action principle," which is the only new material in this section.

Let $\mathcal{X}=\mathbb{Z}^{\mathbb{Z}^{d}}$. We think of elements of $\mathcal{X}$ as particle configurations on $\mathbb{Z}^{d}$ in which some sites may have a negative number of particles. We endow $\mathcal{X}$ with the Borel $\sigma$-algebra coming from the product topology, with $\mathbb{Z}$ having the discrete topology. On $\mathbb{Z}^{d}$ and on $\mathbb{N}$ we use the full power set as a $\sigma$-algebra, and on the half-line $[0, \infty)$ we use the usual Borel $\sigma$-algebra. A toppling procedure is a measurable function

$$
T:[0, \infty) \times \mathbb{Z}^{d} \times \mathcal{X} \rightarrow \mathbb{N}
$$

satisfying for all $\eta \in \mathcal{X}$ and all $x \in \mathbb{Z}^{d}$

(a) $T(0, x, \eta)=0$.

(b) The function $t \rightarrow T(t, x, \eta)$ is right-continuous and nondecreasing with jumps of size at most one, i.e., for all $t \geq 0$,

$$
T(t, x, \eta)-\lim _{s \uparrow t} T(s, x, \eta) \leq 1 .
$$

(c) In every finite time interval, there are only finitely many jumps at $x$.

(d) There is no "infinite backward chain of topplings," i.e., no path $x_{1} \sim x_{2} \sim \cdots$ and sequence of times $t_{1}>t_{2}>\cdots$ such that for all $i=1,2, \ldots$

$$
T\left(t_{i}, x_{i}, \eta\right)>\lim _{s \uparrow t_{i}} T\left(s, x_{i}, \eta\right)
$$

We interpret $T(t, x, \eta)$ as the number of times $x$ topples in the time interval $[0, t]$, for initial configuration $\eta$. We say that $x$ topples at time $t$ for initial configuration $\eta$, if

$$
T(t, x, \eta)>\lim _{s \uparrow t} T(s, x, \eta) .
$$

The toppling procedure $T$ may be deterministic (as in parallel updates or nested volumes) or random (as in Markov toppling). A random toppling procedure can be viewed as a measurable function

$$
T:[0, \infty) \times \mathbb{Z}^{d} \times \mathcal{X} \times \Omega \rightarrow \mathbb{N} \cup\{\infty\}
$$

where $\Omega$ is a probability space. In this case, we require $T(\cdot, \cdot, \cdot, \omega)$ to satisfy properties (a)-(d) for almost all $\omega \in \Omega$. 
If $u, v$ are functions on $\mathbb{Z}^{d}$, write $u \leq v$ if $u(x) \leq v(x)$ for all $x \in \mathbb{Z}^{d}$. The discrete Laplacian $\Delta u$ of $u$ is the function

$$
\Delta u(x)=\sum_{y \sim x} u(y)-2 d u(x)
$$

where the sum is taken over the $2 d$ lattice neighbors of $x$.

Given a toppling procedure $T$ and initial configuration $\eta$, the resulting configuration at time $t$ is

$$
\eta_{t}:=\eta+\Delta T(t, \cdot, \eta) .
$$

We say that $T$ is legal for $\eta$ if for all $x \in \mathbb{Z}^{d}$ and all $t \geq 0$ such that $x$ topples at time $t$, we have

$$
\lim _{s \uparrow t} \eta_{s}(x) \geq 2 d .
$$

That is, in a legal toppling procedure only unstable sites are toppled.

We set

$$
T(\infty, x, \eta)=\sup _{t \geq 0} T(t, x, \eta) \in \mathbb{N} \cup\{\infty\} .
$$

We say that $T$ is finite for initial configuration $\eta$, if $T(\infty, x, \eta)<\infty$ for all $x \in \mathbb{Z}^{d}$. In this case, we say that $T$ is stabilizing for $\eta$ if

$$
\eta_{\infty}:=\eta+\Delta T(\infty, \cdot, \eta) \leq \overline{2 d-1}
$$

That is, in the final configuration $\eta_{\infty}$ no site is unstable.

The following lemma has been proved a number of times in various settings $[2-4,8,11]$.

Lemma 2.1 (Abelian property) For any $\eta \in \mathcal{X}$, if $T$ is a finite legal stabilizing toppling procedure for $\eta$, then any legal toppling procedure is finite for $\eta$. Moreover if $T^{\prime}$ is another legal stabilizing toppling procedure for $\eta$, then for all $x \in \mathbb{Z}^{d}$

$$
T(\infty, x, \eta)=T^{\prime}(\infty, x, \eta)
$$

If $\eta$ is a particle configuration for which there exists a finite legal stabilizing toppling procedure, then we say that $\eta$ stabilizes; otherwise, we say that $\eta$ is exploding. If $\eta$ stabilizes, then the function $u: \mathbb{Z}^{d} \rightarrow \mathbb{N}$ given by

$$
u(x)=T(\infty, x, \eta)
$$

where $T$ is any legal stabilizing toppling procedure for $\eta$, is called the odometer of $\eta$.

Note that if every site topples at least once, then $\eta$ must be exploding. Otherwise, by the no infinite backward chain condition (d), some site $x \in \mathbb{Z}^{d}$ must finish toppling no later than all of its neighbors do; since each neighbor topples at least once more, $x$ receives $2 d$ additional particles and must topple again, a contradiction. Thus we have shown

Lemma 2.2 [8, Theorem 2.8, item 4] If $\eta$ stabilizes, then $u(x)=0$ for some $x \in \mathbb{Z}^{d}$. 
Let $\eta$ be a particle configuration that stabilizes, and let $u$ be its odometer function (2). In our application, we will take $\eta=\sigma+n \delta_{o}$, where $\sigma$ is a robust background. Since $\Delta u(x)$ counts the net number of particles exiting the site $x$, the stabilization $\eta_{\infty}$ of $\eta$ is given by

$$
\eta_{\infty}=\eta+\Delta u
$$

Definition Given a particle configuration $\eta$ on $\mathbb{Z}^{d}$, a function $u_{1}: \mathbb{Z}^{d} \rightarrow \mathbb{Z}$, is called stabilizing for $\eta$ if

$$
\eta+\Delta u_{1} \leq \overline{2 d-1}
$$

Informally, we may think of $\eta+\Delta u_{1}$ as the configuration obtained from $\eta$ by performing $u_{1}(x)$ topplings at each site $x \in \mathbb{Z}^{d}$. Note, however, that the above definition makes no requirement that these topplings be legal; that is, they may produce sites with a negative number of particles.

Our proof of Theorem 1.1 rests on the following lemma, which characterizes the odometer function $u$ as minimal among all nonnegative stabilizing functions. Deepak Dhar has aptly called this a "least action principle," in the sense that the number of topplings in a legal toppling sequence is the minimum number required to stabilize the configuration. In fact, more is true: not only is the total number of topplings minimized, but each vertex does the minimum amount of work required of it to stabilize the configuration.

According to the abelian property, if we use a legal toppling procedure to stabilize $\eta$, then each site $x$ topples exactly $u(x)$ times, regardless of the choice of procedure. The least action principle says that in any sequence of topplings that stabilizes $\eta$, even if some of those topplings are illegal, each site $x$ topples at least $u(x)$ times.

Lemma 2.3 (Least Action Principle) Let $\eta$ be a particle configuration on $\mathbb{Z}^{d}$ that is not exploding, and let $u$ be its odometer. If $u_{1}: \mathbb{Z}^{d} \rightarrow \mathbb{N}$ is stabilizing for $\eta$, then $u_{1} \geq u$.

Proof To compare $u_{1}$ to the odometer, we use the following discrete time legal toppling procedure $T^{\prime}$. Enumerate the sites in $\mathbb{Z}^{d}$. Call a site $x \in \mathbb{Z}^{d}$ ready if it has at least $2 d$ particles and has toppled fewer than $u_{1}(x)$ times. At each time step, if there are any ready sites, topple the smallest ready site.

Write $u^{\prime}(x)=T^{\prime}(\infty, x, \eta)$ for the number of times $x$ topples during this procedure. We will show that $u^{\prime}=u$. If $\eta^{\prime}=\eta+\Delta u^{\prime}$ is stable, then $T^{\prime}$ is stabilizing as well as legal, so $u^{\prime}=u$ by the abelian property. Otherwise, $\eta^{\prime}$ has some unstable site $y$. We must have $u^{\prime}(y)=u_{1}(y)$; otherwise, $y$ would still be ready. Writing $u^{\prime \prime}=u_{1}-u^{\prime}$, we obtain

$$
\left(\eta+\Delta u_{1}\right)(y)=\eta^{\prime}(y)+\Delta u^{\prime \prime}(y) \geq \eta^{\prime}(y) \geq 2 d
$$

since $u^{\prime \prime}(y)=0$. This contradicts the assumption that $u_{1}$ is stabilizing.

We pause here to record a closely related fact. If $u_{1}, u_{2}$ are functions on $\mathbb{Z}^{d}$, write $\min \left(u_{1}, u_{2}\right)$ for their pointwise minimum. If $x \in \mathbb{Z}^{d}$ is a site where $u_{1}(x) \leq u_{2}(x)$, then

$$
\begin{aligned}
\Delta \min \left(u_{1}, u_{2}\right)(x) & =\sum_{y \sim x} \min \left(u_{1}(y), u_{2}(y)\right)-2 d u_{1}(x) \\
& \leq \sum_{y \sim x} u_{1}(y)-2 d u_{1}(x)=\Delta u_{1}(x) .
\end{aligned}
$$


Likewise, if $u_{1}(x)>u_{2}(x)$, then $\Delta \min \left(u_{1}, u_{2}\right)(x) \leq \Delta u_{2}(x)$. So

$$
\Delta \min \left(u_{1}, u_{2}\right) \leq \max \left(\Delta u_{1}, \Delta u_{2}\right) .
$$

As a consequence, we obtain the following.

Lemma 2.4 If $u_{1}$ and $u_{2}$ are stabilizing for $\sigma$, then $\min \left(u_{1}, u_{2}\right)$ is also stabilizing for $\sigma$.

Proof $\sigma+\Delta \min \left(u_{1}, u_{2}\right) \leq \max \left(\sigma+\Delta u_{1}, \sigma+\Delta u_{2}\right) \leq \overline{2 d-1}$.

The set of stabilizing functions is also closed under adding any constant function, giving it the structure of a module over the tropical semiring $(\mathbb{Z}$, min, +$)$. A related module is studied in [9].

\section{Growth Rates}

Fix an integer $h \leq 2 d-2$, and let $\eta$ be the configuration $\bar{h}+n \delta_{o}$ on $\mathbb{Z}^{d}$. Let $S_{n}$ be the set of sites that ever topple or receive a particle during the stabilization of $\eta$ (in [10] these were called "visited" sites). Note that if $y$ receives a particle, then one of its neighbors must have toppled. Thus $S_{n}$ is related to the set $T_{n}$ of sites that topple by

$$
S_{n}=T_{n} \cup \partial T_{n}
$$

where for $A \subset \mathbb{Z}^{d}$ we write

$$
\partial A=\left\{y \in \mathbb{Z}^{d}: y \notin A, \exists z \in A, z \sim y\right\} .
$$

Write $|x|=\left(x_{1}^{2}+\cdots+x_{d}^{2}\right)^{1 / 2}$ for the Euclidean norm on $\mathbb{Z}^{d}$, and for $r>0$ let

$$
B_{r}=\left\{x \in \mathbb{Z}^{d}:|x|<r\right\}
$$

be the ball of radius $r$ centered at the origin in $\mathbb{Z}^{d}$. Let $\omega_{d}$ be the volume of the unit ball in $\mathbb{R}^{d}$. For the proof of Theorem 1.1, we take as a starting point the following result of [10].

Theorem 3.1 [10, Theorem 4.1] Fix an integer $h \leq 2 d-2$. For any $n \geq 1$, we have

$$
B_{c_{1} r-c_{2}} \subset S_{n}
$$

where $r$ is such that $n=\omega_{d} r^{d}$, and

$$
c_{1}=(2 d-1-h)^{-1 / d}
$$

and $c_{2}$ is a constant depending only on $d$. Moreover if $h \leq d-1$, then for any $n \geq 1$ and any $\epsilon>0$ we have

$$
S_{n} \subset B_{c_{1}^{\prime} r+c_{2}^{\prime}}
$$

where

$$
c_{1}^{\prime}=(d-\epsilon-h)^{-1 / d}
$$

and $c_{2}^{\prime}$ is independent of $n$ but may depend on $d, h$ and $\epsilon$. 
Note that $h$ may be negative, in which case the background $\bar{h}$ corresponds to each site in $\mathbb{Z}^{d}$ starting with a "hole" of depth $H=-h$.

We are grateful to Haiyan Liu for pointing out a gap in the proof of the outer bound (3). The gap occurs in Lemma 4.2 of [10], which is valid only for $H \geq 0$. We correct this gap in Sect. 3.1. Next, in Sect. 3.2, we explain our technique of "background modification," and use it to deduce Theorem 1.1 from Theorem 3.1.

\subsection{Low Background Height}

Fix $0 \leq h \leq d-1$, let $\eta$ be the configuration $\bar{h}+n \delta_{o}$ on $\mathbb{Z}^{d}$, and consider the odometer function

$$
u_{n}(x)=\text { number of times } x \text { topples during the stabilization of } \eta \text {. }
$$

The normalization of the odometer function and of the discrete Laplacian (1) differs by a factor of $2 d$ from the one used in [10]. It is the most convenient normalization for the abelian sandpile, since $2 d$ particles move in every toppling.

In [10] it is proved that for every site $x \in \mathbb{Z}^{d}$ with $c_{1}^{\prime} r-1<|x| \leq c_{1}^{\prime} r$ we have

$$
u_{n}(x) \leq c
$$

where $c$ is a constant which may depend on $d, h$ and $\epsilon$ but not on $n$. (In the notation of [10], $c=c_{2}^{\prime} / 2 d$.)

It follows that $u_{n}$ is uniformly bounded outside the ball $B_{c_{1}^{\prime} r}$; indeed, if $|x|>c_{1}^{\prime} r$, then setting $n^{\prime}=\left\lceil\omega_{d}\left(|x| / c_{1}^{\prime}\right)^{d}\right\rceil$, since $n \leq n^{\prime}$ we have by the abelian property

$$
u_{n}(x) \leq u_{n^{\prime}}(x) \leq c
$$

The next lemma shows that in fact, $u_{n}=0$ outside the slightly larger ball $B_{c_{1}^{\prime} r+c-1}$. Hence $T_{n} \subset B_{c_{1}^{\prime} r+c-1}$, and hence $S_{n} \subset B_{c_{1}^{\prime} r+c}$, which completes the proof of (3).

Lemma 3.2 For all $j=0,1, \ldots, c$ and all $x \in \mathbb{Z}^{d}$ with $|x|>c_{1}^{\prime} r+j-1$, we have

$$
u_{n}(x) \leq c-j
$$

Proof Let

$$
R_{j}=\left\{x \in \mathbb{Z}^{d}:|x|>c_{1}^{\prime} r+j-1\right\} .
$$

Note that for any $x \in R_{j}$, all neighbors $y \sim x$ lie in $R_{j-1}$, and at least $d$ neighbors have $|y| \geq|x|$, so at least $d$ neighbors lie in $R_{j}$.

We will prove the lemma by induction on $j$. Let

$$
U_{j}=\left\{x \in R_{j} \mid u_{n}(x)>c-j\right\} .
$$

If $U_{j}$ is empty, then the proof is complete. Otherwise, by the no infinite backward chain condition, there exists a site $x \in U_{j}$ that finishes toppling no later than all of its neighbors in $U_{j}$. By the inductive hypothesis, every neighbor $y$ of $x$ satisfies.

$$
u_{n}(y) \leq c-j+1 \text {. }
$$


Just before $x$ topples for the last time, each neighbor $y \in U_{j}$ has not yet toppled for the last time, so $y$ has toppled at most $c-j$ times. Moreover, each neighbor $y \in R_{j}-U_{j}$ has toppled at most $c-j$ times; and each neighbor $y \notin R_{j}$ has toppled at most $c-j+1$ times. Hence, just before it topples for the last time, $x$ has received at most $d(c-j)+d(c-j+1)$ chips and emitted at least $2 d(c-j)$ chips, leaving it with at most $h+d$ chips. Since $h \leq d-1$, this is not enough chips to topple, which gives the required contradiction.

\subsection{High Background Height}

To prove Theorem 1.1 using the least action principle (Lemma 2.3), for each coordinate $i=1, \ldots, d$ we will construct a toppling function $g_{i}$ supported in the slab

$$
A_{i, r}=\left\{x \in \mathbb{Z}^{d}:\left|x_{i}\right| \leq r\right\}
$$

The effect of toppling according to $g_{i}$ will be to modify the constant background height $h$ by "clearing out" particles down to height at most $d-1$ in a smaller slab $A_{i, r_{0}}$ and "piling them up" to height at most $2 d-1$ outside $A_{i, r_{0}}$. We will see that this can be done while keeping $r_{0}$ proportional to $r$.

On this modified background, $n$ particles at the origin will spread with a growth rate at most according to $h=d-1$, provided $n$ is small enough so that the particles do not spread outside $Q_{r_{0}}$. This growth rate is controlled by Theorem 3.1: $n$ particles on constant background height $d-1$ in $\mathbb{Z}^{d}$ spread at most a distance of order $n^{1 / d}$. Since $r_{0}$ is proportional to $r$, we can therefore choose $n$ proportional to $r^{d}$.

The desired background modification can be accomplished a function of just one coordinate, $g_{i}\left(x_{1}, \ldots, x_{d}\right)=g\left(x_{i}\right)$. The next lemma spares us the need to specify $g$ explicitly; it suffices to specify how the background is modified. In the lemma, $g$ plays the role of toppling function on $\mathbb{Z}$, and $f$ represents the net change in height of the configuration. The conditions (5) mean in words that topplings cannot change the total number of particles, nor the center of mass of a configuration.

Lemma 3.3 If $f: \mathbb{Z} \rightarrow \mathbb{Z}$ is supported on a finite interval $I=[-a, b]$, and

$$
\sum_{y \in I} f(y)=\sum_{y \in I} y f(y)=0
$$

then $f=\Delta g$ for an integer-valued function $g$ supported on the interval $I^{\prime}=[1-a, b-1]$. Moreover, if there are no $x_{1}<x_{2}<x_{3}$ such that $f\left(x_{1}\right)<0, f\left(x_{2}\right)>0$ and $f\left(x_{3}\right)<0$, then $g \geq 0$.

Proof Let

$$
g(x)=\sum_{y=-a}^{x-1}(x-y) f(y) .
$$

Then for $x \geq b$ we have

$$
g(x)=x \sum_{y=-a}^{b} f(y)-\sum_{y=-a}^{b} y f(y)=0
$$


so $g$ is supported on $I^{\prime}$. Also

$$
\begin{aligned}
\Delta g(x) & =g(x+1)-2 g(x)+g(x-1) \\
& =f(x)+\sum_{y=-a}^{x-2}((x+1-y)-2(x-y)+(x-1-y)) f(y)=f(x)
\end{aligned}
$$

as desired.

If $g(z)<0$ for some $z$, then since $g(-a)=g(b)=0$, the difference

$$
D g(y)=g(y+1)-g(y)
$$

satisfies $D g\left(y_{1}\right)<0$ and $D g\left(y_{2}\right)>0$ for some $y_{1}<z \leq y_{2}$. Hence the second difference

$$
f(x)=\Delta g(x)=D g(x)-D g(x-1)
$$

satisfies $f\left(x_{1}\right)<0, f\left(x_{2}\right)>0$ and $f\left(x_{3}\right)<0$ for some $x_{1} \leq y_{1}<x_{2} \leq y_{2}<x_{3}$.

Proof of Theorem 1.1 For each $i=1, \ldots, d$ we will construct a nonnegative function $u_{i}$ on $\mathbb{Z}^{d}$ which is stabilizing for the configuration $\bar{h}+n \delta_{o}$, and supported on the infinite slab $A_{i, r}$; see (4).

By the least action principle, Lemma 2.3, the odometer function $u$ satisfies $u \leq u_{i}$ for $i=1, \ldots, d$. Since $T_{n, d, \bar{h}}$ is the support of $u$, we obtain

$$
T_{n, d, \bar{h}} \subseteq \bigcap_{i=1}^{d} A_{i, r}=Q_{r} .
$$

To construct $u_{i}$, let $w: \mathbb{Z}^{d} \rightarrow \mathbb{N}$ be the odometer function for the configuration $\overline{d-1}+$ $n \delta_{o}$. By Theorem 3.1, if $n$ is sufficiently large, then $w$ is supported on the ball centered at the origin of radius

$$
\rho=\left(1+\frac{\epsilon}{2 d}\right)\left(\frac{n}{\omega_{d}}\right)^{1 / d} .
$$

In particular, $w$ vanishes outside the cube $Q_{\rho}$.

Let $r_{0}$ be the smallest integer multiple of $2 d-1-h$ exceeding $\rho$, and let

$$
r_{1}=\frac{d}{2 d-1-h} r_{0}
$$

Let $f: \mathbb{Z} \rightarrow \mathbb{Z}$ be given by

$$
f(x)= \begin{cases}2(d-1-h), & x=0, \\ d-1-h, & 0<|x|<r_{0}, \\ 2 d-1-h, & r_{0} \leq|x|<r_{1}, \\ 0, & |x| \geq r_{1}\end{cases}
$$

Then with $I=\left[1-r_{1}, r_{1}-1\right]$

$$
\begin{aligned}
\sum_{y \in I} f(y) & =2 r_{0}(d-1-h)+\left(2 r_{1}-2 r_{0}\right)(2 d-1-h) \\
& =-2 d r_{0}+2(2 d-1-h) r_{1}=0 .
\end{aligned}
$$


Since $f(y)=f(-y)$ we have $\sum_{y \in I} y f(y)=0$. By Lemma 3.3, $f=\Delta g$ for a nonnegative integer-valued function $g$ supported on the interval $I^{\prime}=\left[2-r_{1}, r_{1}-2\right]$.

For $x=\left(x_{1}, \ldots, x_{d}\right) \in \mathbb{Z}^{d}$, define

$$
u_{i}(x)=w(x)+g\left(x_{i}\right) .
$$

Note that the function $g\left(x_{i}\right)$ has $d$-dimensional Laplacian $f\left(x_{i}\right)$. Inside the cube $Q_{\rho}$, we have $f\left(x_{i}\right) \leq d-1-h$, hence inside $Q_{\rho}$

$$
h+n \delta_{o}+\Delta u_{i} \leq d-1+n \delta_{o}+\Delta w \leq 2 d-1 .
$$

Outside $Q_{\rho}$, since $w$ vanishes and $f\left(x_{i}\right) \leq 2 d-1-h$, we have

$$
h+n \delta_{o}+\Delta u_{i} \leq 2 d-1 \text {. }
$$

Thus $u_{i}$ is stabilizing for $h+n \delta_{o}$. Moreover, since

$$
\begin{aligned}
r_{1} & \leq d\left(\frac{\rho}{2 d-1-h}+1\right) \\
& =\frac{d+\epsilon / 2}{2 d-1-h}\left(\frac{n}{\omega_{d}}\right)^{1 / d}+d
\end{aligned}
$$

we have $r_{1} \leq r$ for sufficiently large $n$, hence $u_{i}$ is supported on the slab $A_{i, r}$ as desired.

We remark that in addition to bounding the set of sites $T_{n, d, \bar{h}}$ that topple, the proof gives a bound on the odometer function

$$
u(x)=\# \text { times } x \text { topples in the stabilization of } \bar{h}+n \delta_{o} \text { in } \mathbb{Z}^{d},
$$

namely

$$
\begin{aligned}
u(x) & \leq \min \left(u_{1}(x), \ldots, u_{d}(x)\right) \\
& =w(x)+\min \left(g\left(x_{1}\right), \ldots, g\left(x_{d}\right)\right) \\
& =w(x)+g\left(\max \left|x_{i}\right|\right) .
\end{aligned}
$$

By Lemma 2.4, the right side is stabilizing for $\bar{h}+n \delta_{o}$. The resulting stable configuration in the case $d=h=2$ is pictured in Fig. 3 .

The proof of Theorem 1.2 is identical to that of Theorem 1.1, except that we now choose

$$
f(x)= \begin{cases}2-2 d, & x=0, \\ -d, & 0<|x|<r_{0} \text { and } m \nmid x, \\ 1-d, & 0<|x|<r_{0} \text { and } m \mid x, \\ 0, & r_{0} \leq|x|<r_{1} \text { and } m \nmid x, \\ 1, & r_{0} \leq|x|<r_{1} \text { and } m \mid x, \\ 0, & |x| \geq r_{1},\end{cases}
$$

where $r_{0}$ the smallest integer exceeding $\rho=\left(1+\frac{\epsilon}{2 d}\right)\left(\frac{n}{\omega_{d}}\right)^{1 / d}$, and

$$
r_{1}=m\left(d r_{0}-1\right) \text {. }
$$



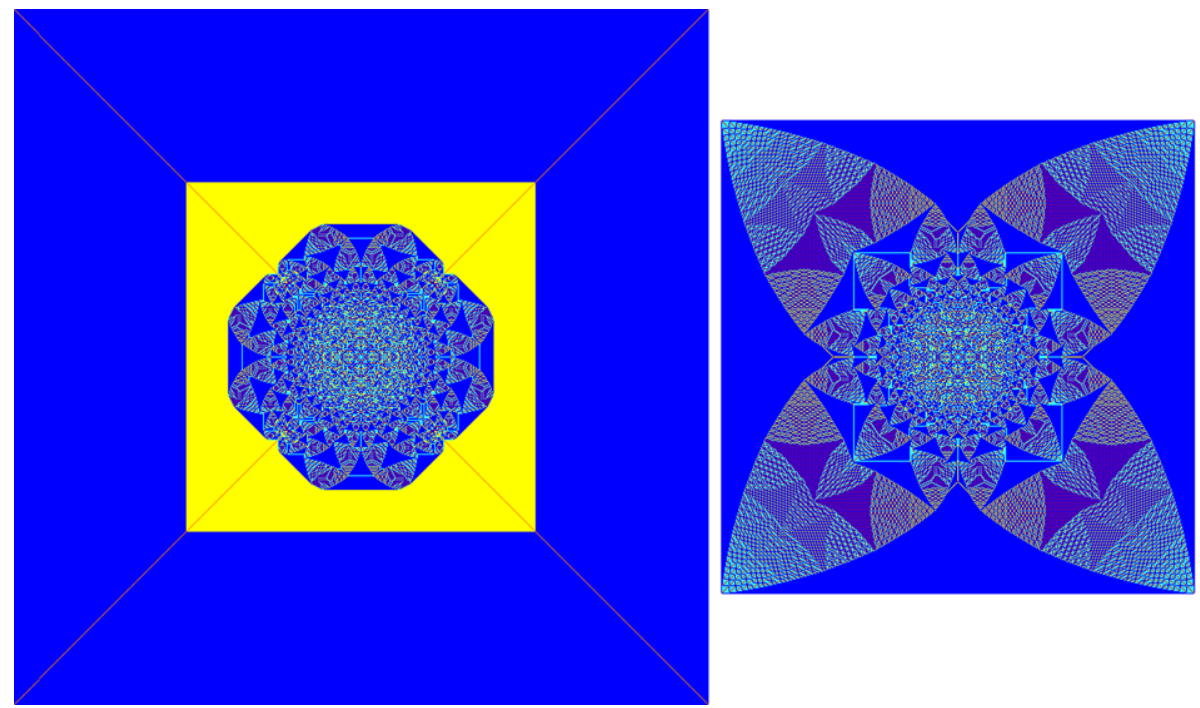

Fig. 3 (Color online) Left: The stable configuration $\overline{2}+n \delta_{o}+\Delta \min \left(u_{1}, u_{2}\right)$ constructed in the proof of Theorem 1.1. Sites with negative height, along the diagonals of the square, are colored orange. Right: The stabilization of $\overline{2}+n \delta_{o}$. Here $n=10^{5}$

Then we have that

$$
r_{1} \leq m\left(d+\frac{\epsilon}{2}\right)\left(\frac{n}{\omega_{d}}\right)^{1 / d}+m(d-1),
$$

so that again $r_{1} \leq r$ for sufficiently large $n$.

\section{Robust and Explosive Backgrounds}

Write $\psi_{1}=e_{1}, \ldots, \psi_{d}=e_{d}, \psi_{d+1}=-e_{1}, \ldots, \psi_{2 d}=-e_{d}$ for the $2 d$ coordinate directions in $\mathbb{Z}^{d}$. If $R$ is a rectangular prism in $\mathbb{Z}^{d}$, write

$$
F_{i}(R)=\left\{y: y \notin R, y-\psi_{i} \in R\right\}
$$

for the outer face of $R$ in direction $\psi_{i}$.

We will deduce Propositions 1.3 and 1.4 from the following slightly more general result.

Theorem 4.1 Let $\sigma$ be a background on $\mathbb{Z}^{d}$ satisfying

(i) $\sigma(x) \geq 2 d-2$ for all $x \in \mathbb{Z}^{d}$; and

(ii) There exists $r_{0} \in \mathbb{N}$ such that for all $r \geq r_{0}$, each outer face $F_{i}\left(Q_{r}\right)$ contains a site $x$ with $\sigma(x) \geq 2 d-1$.

Then $\sigma$ is explosive.

Proof By Lemma 2.2, in order to prove that a configuration $\eta$ on $\mathbb{Z}^{d}$ is exploding, it suffices to find a toppling procedure in which every site in $\mathbb{Z}^{d}$ topples at least once. 
From [6, Theorem 4.1], if the background height is exactly $2 d-2$, then for every $n$, the set of sites that topple during stabilization forms a cube $Q_{r}$, and we can choose $n$ so that $r \geq r_{0}$.

Let $R_{0}=Q_{r}$ and

$$
R_{k}=R_{k-1} \cup F_{k \bmod 2 d}\left(R_{k-1}\right), \quad k \geq 1 .
$$

We will define a toppling order in stages $k=0,1,2, \ldots$ so that at the end of stage $k$, all sites in $R_{k}$ have toppled at least once, and no other sites have toppled. Since $\bigcup_{k \geq 0} R_{k}=\mathbb{Z}^{d}$, it follows that every site in $\mathbb{Z}^{d}$ topples at least once, so $\sigma+n \delta_{o}$ is exploding.

During stage 0 , we perform all the topplings that occur in the stabilization of $\overline{2 d-2}+$ $n \delta_{o}$. Then every site in the cube $R_{0}$ has toppled at least once, and no other sites have toppled. Hence by (i), every site in every outer face of $R_{0}$ now has at least $2 d-1$ particles, and by (ii), in every outer face there is at least one unstable site.

The remaining stages are defined inductively. After stage $k-1$, every site in $F=$ $F_{k \bmod 2 d}\left(R_{k-1}\right)$ has at least $2 d-1$ particles, and at least one site $y \in F$ is unstable. Topple first $y$, then its neighbors in $F$, then the sites in $F$ at distance 2 from $y$, and so on, until all sites in $F$ have toppled once. Now every site in $R_{k}$ has toppled at least once, and no sites outside $R_{k}$ have toppled, completing the inductive step.

To deduce Proposition 1.3 from Theorem 4.1, since

$$
\operatorname{gcd}\left(x_{1 j}, \ldots, x_{d j}\right)=1, \quad j=1, \ldots, d
$$

there exist integers $a_{i j}$ for $1 \leq i, j \leq d$, satisfying

$$
\sum_{i=1}^{d} a_{i j} x_{i j}=1, \quad j=1, \ldots, d .
$$

Then for each $j=1, \ldots, d$, the vector

$$
\mathbf{v}_{j}=\sum_{i=1}^{d} a_{i j} \mathbf{x}_{i} \in L
$$

has $e_{j}$-coordinate $v_{j j}=1$, so any hyperplane in $\mathbb{Z}^{d}$ parallel to one of the coordinate hyperplanes intersects $L$. Moreover, $L$ contains the vectors $D e_{j}$ for $j=1, \ldots, d$, where $D=\operatorname{det}\left(x_{i j}\right)_{i, j=1}^{d} \neq 0$. Thus $L$ intersects every face $F_{i}\left(Q_{r}\right)$ when $r \geq|D| / 2$.

To deduce Proposition 1.4, it remains to check that the configuration $\overline{2 d-2}+\beta$ on $\mathbb{Z}^{d}$ satisfies condition (ii) of Theorem 4.1 with probability 1 . Write $\mathcal{E}_{i, r}$ for the event that $\beta(x)=0$ for all $x \in F_{i}\left(Q_{r}\right)$. By the independence of the Bernoulli random variables $\beta(x)$, this event has probability

$$
\mathbb{P}\left(\mathcal{E}_{i, r}\right)=(1-\epsilon)^{\left|F_{i}\left(Q_{r}\right)\right|} \leq(1-\epsilon)^{r} .
$$

In particular, $\sum_{r \geq 1} \sum_{i=1}^{2 d} \mathbb{P}\left(\mathcal{E}_{i, r}\right)<\infty$. By the Borel-Cantelli lemma, with probability 1 only finitely many of the events $\mathcal{E}_{i, r}$ occur. We remark on the similarity between this argument and Straley's argument for bootstrap percolation [5].

We define the box $\mathcal{B}_{r}$ as

$$
\mathcal{B}_{r}=\partial Q_{r}=\bigcup_{i=1}^{2 d} F_{i}\left(Q_{r}\right) .
$$


The following theorem is a partial converse to Theorem 4.1, and gives a counterexample to Remark 7.1 in [7].

Theorem 4.2 Let $r_{1}, r_{2}, \ldots$ be an increasing sequence of positive integers. Let $\sigma$ be a stable background on $\mathbb{Z}^{d}$ in which every site in $\mathcal{B}_{r_{1}} \cup \mathcal{B}_{r_{2}} \cup \ldots$ has at most $2 d-2$ particles.

Then $\sigma$ is robust.

The proof uses the following lemma, which follows from [6, Lemma 4.2] and the abelian property.

Lemma 4.3 If $\sigma$ is a stable background satisfying $\sigma(x) \leq 2 d-2$ for all $x \in \mathcal{B}_{r}$, then no sites outside $Q_{r}$ topple during the stabilization of $\sigma+\delta_{o}$.

Proof of Theorem 4.2 We need to show that $\sigma+n \delta_{o}$ stabilizes in finitely many topplings, for every $n \in \mathbb{N}$. We induct on $n$ to show that no sites outside $Q_{r_{n}}$ topple during the stabilization of $\sigma+n \delta_{o}$.

By Lemma 4.3, no sites outside $Q_{r_{1}}$ topple during the stabilization of $\sigma+\delta_{o}$.

Let $\sigma_{n}$ be the stabilization of $\sigma+n \delta_{o}$. By the inductive hypothesis, no sites topple outside $Q_{r_{n}}$ during this stabilization, so $\sigma_{n}(x) \leq 2 d-2$ for all $x \in \mathcal{B}_{r_{n+1}}$. By Lemma 4.3, no sites outside $Q_{r_{n+1}}$ topple during the stabilization of $\sigma_{n}+\delta_{o}$. By the abelian property, a site topples during the stabilization of $\sigma+(n+1) \delta_{o}$ if and only if it topples during the stabilization of $\sigma+n \delta_{o}$ or during the stabilization of $\sigma_{n}+\delta_{o}$. This completes the inductive step.

Remark Theorem 4.2 remains true for arbitrary disjoint rectangular boxes surrounding the origin; they need not be cubical or centered at the origin.

\section{Dimensional Reduction}

Our argument used properties of the one-dimensional sandpile to bound the growth rate of higher-dimensional sandpiles. There appears to be a deeper relationship between sandpiles in $d$ and $d-1$ dimensions, which we formulate in the following dimensional reduction conjecture. For $x \in \mathbb{Z}^{d}$, let $\sigma_{n, d}(x)$ be the final number of particles present at $x$ in the stabilization of $\overline{2 d-2}+n \delta_{o}$. Write $\operatorname{rad}(n, d)$ for the radius of the cube $\left\{x \in \mathbb{Z}^{d} \mid \sigma_{n, d}(x)>0\right\}$. Note that by Theorem $1.1, \operatorname{rad}(n, d)$ has order $n^{1 / d}$.

Identifying $\mathbb{Z}^{d-1}$ with the hyperplane $x_{d}=0$ in $\mathbb{Z}^{d}$, we would like to compare the slice through the origin $Q_{\operatorname{rad}(n, d)} \cap \mathbb{Z}^{d-1}$ of the $d$-dimensional sandpile started from $n$ particles with a $(d-1)$-dimensional sandpile started from some number $m$ of particles. Given $m, n$ and $d$, let us call a site $x=\left(x_{1}, \ldots, x_{d-1}\right) \in \mathbb{Z}^{d-1}$ an exact match if

$$
\sigma_{n, d}\left(x_{1}, \ldots, x_{d-1}, 0\right)=2+\sigma_{m, d-1}\left(x_{1}, \ldots, x_{d-1}\right) \text {. }
$$

Given $0<\lambda<1$, consider the subset $A_{\lambda}$ of the slice through the origin

$$
A_{\lambda}=\left(Q_{\operatorname{rad}(n, d)}-Q_{\lambda \operatorname{rad}(n, d)}\right) \cap \mathbb{Z}^{d-1} .
$$

Conjecture 5.1 There exists a constant $\lambda=\lambda_{d}<1$ such that for all $n \geq 1$ there exists $m \geq 1$ such that all but $O\left(\operatorname{rad}(n, d)^{d-2}\right)$ sites in $A_{\lambda}$ are exact matches. 

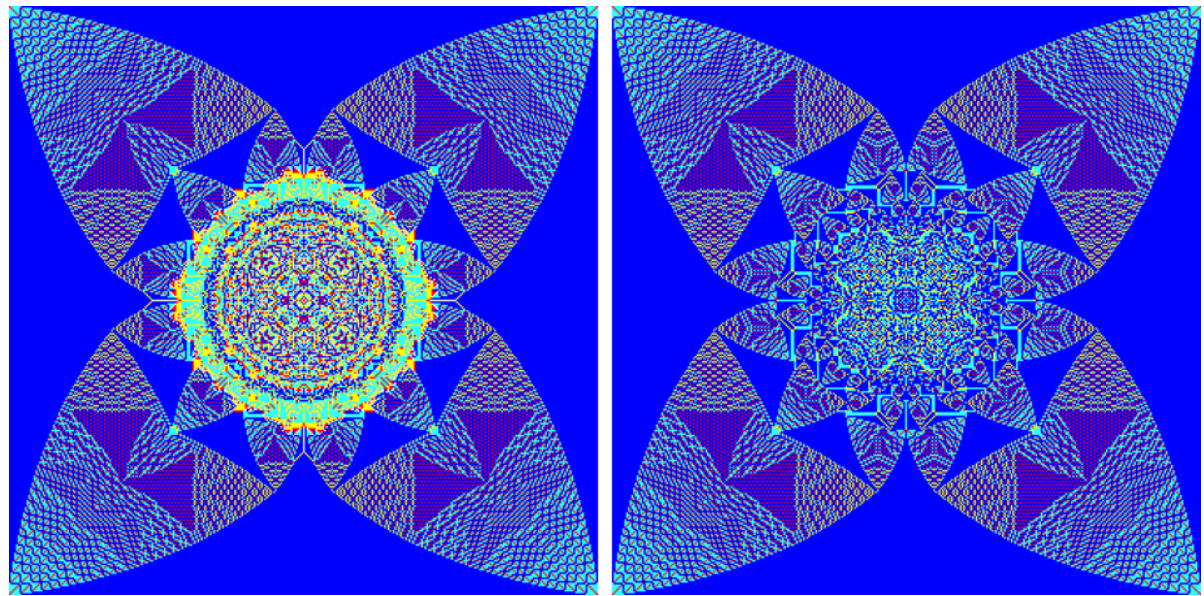

Fig. 4 (Color online) Left: A two-dimensional slice through the origin of the sandpile of $n=5 \cdot 10^{6}$ particles in $\mathbb{Z}^{3}$ on background height $h=4$. Right: The sandpile of $m=47465$ particles in $\mathbb{Z}^{2}$ on background height $h=2$. Color scheme on left: sites colored blue have 5 particles, turquoise 4 , yellow 3, red 2, gray 1 , white 0 . On right: blue 3 particles, turquoise 2, yellow 1 , red 0

The case $d=3$ is illustrated in Fig. 4. Amazingly, except in a region near the origin, the two pictures shown in the figure agree pixel for pixel. For some rare values of $n$, certain small "defects" or "filaments" in the two pictures fail to match exactly, which is why we exclude up to $O\left(\operatorname{rad}(n, d)^{d-2}\right)$ sites. For simplicity, we have restricted our formulation to the slice through the origin, but dimensional reduction seems to occur in all slices except for those close to the boundary of the cube. The value of $m$ is the same for all of these slices. We first learned of the dimensional reduction phenomenon from Deepak Dhar.

Open Access This article is distributed under the terms of the Creative Commons Attribution Noncommercial License which permits any noncommercial use, distribution, and reproduction in any medium, provided the original author(s) and source are credited.

\section{References}

1. Bak, P., Tang, C., Wiesenfeld, K.: Self-organized criticality: an explanation of the $1 / f$ noise. Phys. Rev. Lett. 59(4), 381-384 (1987)

2. Björner, A., Lovász, L., Shor, P.: Chip-firing games on graphs. Eur. J. Comb. 12(4), $283-291$ (1991)

3. Dhar, D.: Self-organized critical state of sandpile automaton models. Phys. Rev. Lett. 64, 1613 (1990)

4. Diaconis, P., Fulton, W.: A growth model, a game, an algebra, Lagrange inversion, and characteristic classes. Rend. Semin. Mat. Univ. Pol. Torino 49(1), 95-119 (1991)

5. van Enter, A.C.D.: Proof of Straley's argument for bootstrap percolation. J. Stat. Phys. 48, 943-945 (1987)

6. Fey, A., Redig, F.: Limiting shapes for deterministic centrally seeded growth models. J. Stat. Phys. 130(3), 579-597 (2008)

7. Fey-den Boer, A., Redig, F.: Organized versus self-organized criticality in the abelian sandpile model. Markov Processes Relat. Fields 11(3), 425-442 (2005)

8. Fey-den Boer, A., Meester, R., Redig, F.: Stabilizability and percolation in the infinite volume sandpile model. Ann. Probab. 37(2), 654-675 (2009) 
9. Haase, C., Musiker, G., Yu, J.: Linear systems on tropical curves (2009). http://arxiv.org/abs/0909.3685

10. Levine, L., Peres, Y.: Strong spherical asymptotics for rotor-router aggregation and the divisible sandpile. Potential Anal. 30, 1-27 (2009)

11. Meester, R., Redig, F., Znamenski, D.: The abelian sandpile model, a mathematical introduction. Markov Processes Relat. Fields 7, 509-523 (2001) 